\title{
Evaluating the effects of feedback type on older adults' performance in mid-air pointing and target selection
}

Conference or Workshop Item

Accepted Version

Theil Cabreira, A. and Hwang, F. (2018) Evaluating the effects of feedback type on older adults' performance in mid-air pointing and target selection. In: SUI '18 The 6th ACM Symposium on Spatial User Interaction, 13-14 Oct 2018, Berlin, Germany, pp. 111-119. (ISBN: 9781450357081) Available at http://centaur.reading.ac.uk/78994/

It is advisable to refer to the publisher's version if you intend to cite from the work. See Guidance on citing.

Published version at: https://doi.org/10.1145/3267782.3267933

All outputs in CentAUR are protected by Intellectual Property Rights law, including copyright law. Copyright and IPR is retained by the creators or other copyright holders. Terms and conditions for use of this material are defined in 
the End User Agreement.

www.reading.ac.uk/centaur

\section{CentAUR}

Central Archive at the University of Reading

Reading's research outputs online 


\title{
Evaluating the Effects of Feedback Type on Older Adults' Performance in Mid-Air Pointing and Target Selection
}

\author{
Full Paper ${ }^{\dagger}$
}

\author{
Arthur Theil Cabreira \\ University of Reading \\ RG6-6AY \\ United Kingdom \\ a.theilcabreira@pgr.reading.ac.uk
}

\author{
Faustina Hwang \\ University of Reading \\ RG6-6AY \\ United Kingdom \\ f.hwang@reading.ac.uk
}

\begin{abstract}
"Hands-free" pointing techniques used in mid-air gesture interaction require precise motor control and dexterity. Although being applied in a growing number of interaction contexts over the past few years, this input method can be challenging for older users $(60+$ years old $)$ who experience natural decline in pointing abilities due to natural ageing process. We report the findings of a target acquisition experiment in which older adults had to perform "pointand-select" gestures in mid-air. The experiment investigated the effect of 6 feedback conditions on pointing and selection performance of older users. Our findings suggest that the bimodal combination of Visual and Audio feedback lead to faster target selection times for older adults, but did not lead to making less errors. Furthermore, target location on screen was found to play a more important role in both selection time and accuracy of pointand-select tasks than feedback type.
\end{abstract}

\section{CCS CONCEPTS}

- Human-centered computing $\rightarrow$ Human computer interaction $(\mathrm{HCI}) \rightarrow$ Interaction techniques $\rightarrow$ Pointing

\section{KEYWORDS}

Aging; Leap Motion gesture-based interfaces; freehand interaction

ACM Reference format:

A. T. Cabreira and F. Hwang. 2018. Evaluating the Effects of Feedback Type on Older Adults' Performance in Mid-Air Pointing and Target Selection. In Proceedings of the 6th Symposium on Spatial User Interaction, Berlin, Germany, October 2018 (SUI'18), 9 pages.

https://doi.org/10.1145/3267782.3267933

Permission to make digital or hard copies of all or part of this work for personal or classroom use is granted without fee provided that copies are not made or distributed for profit or commercial advantage and that copies bear this notice and the full citation on the first page. Copyrights for components of this work owned by others than the author(s) must be honored. Abstracting with credit is permitted. To copy otherwise, or republish, to post on servers or to redistribute to lists, requires prior specific permission and/or a fee. Request permissions from Permissions@acm.org.

SUI '18, October 13-14, 2018, Berlin, Germany

(C) 2018 Copyright is held by the owner/author(s). Publication rights licensed to ACM.

ACM ISBN 978-1-4503-5708-1/18/10 ..\$15.00

https://doi.org/10.1145/3267782.3267933

\section{INTRODUCTION}

Mid-air gesture interaction has been applied in a diverse range of interface applications over the past few years, mostly as a result of the growing number of currently existing motion sensing devices available to the general public such as the Microsoft Kinect, Leap Motion controller, Myo Armband, and the Microsoft Hololens. Despite the variety of gestures supported by those sensor-based devices, point-based interaction is currently the overall most used input technique in mid-air across platforms [6]. That is, users need to move their fingers or hands in mid-air (as a pointer) in order to select interface elements whilst sensors track their physical movements and translate into 2D or 3D coordinates on screen (Figure 1). Tracking accuracy varies across devices but the pointto-select interaction paradigm remains the same. For instance, the Microsoft Kinect tracks hand movements as a single pointer [35] whilst the Leap Motion controller is able to track not only the hand trajectory but also individual fingers [36].

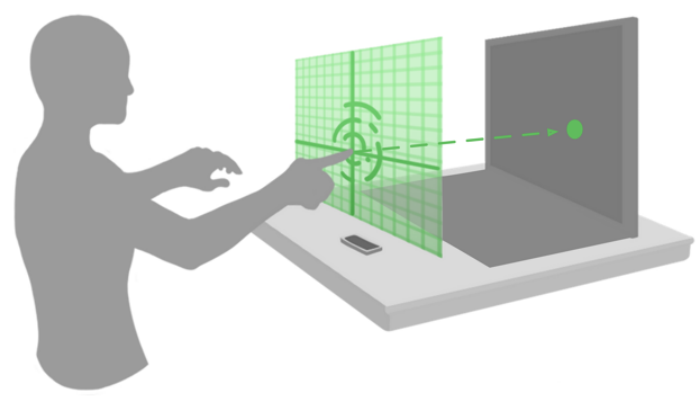

Figure 1: Point-to-select interaction paradigm example.

As mid-air gesture interaction becomes more present in different interaction contexts and this "hands-free" point-based input technique reaches a greater population, the need of assessing its suitability to a broad range of users is still needed. Successful use of pointing techniques requires precise motor control and dexterity $[8,17]$. Using point-to-select input techniques can be a challenge and become a burden for users that experience temporary, sporadic 
or gradual changes in pointing abilities due to ageing, physical impairments, or other situational conditions [5].

The number of older adults (aged 60 and older) has tripled since the year of 1950 and it is estimated that the older population will reach over two billions worldwide by the year of 2050 [3, 30]. However, little research has looked into older adults' needs and expectations for mid-air interaction and point-to-select input techniques. Older adults are known to experience age-related decline in motor, cognitive and sensory abilities that can affect their daily tasks, including the way they interact with technology $[3,8,24]$. Older adults experience natural changes in motor dexterity and muscular strength that may affect pointing abilities due to loss of hand mobility and decreased range of motion $[18,30]$. If these physical limitations are not anticipated by the system design, then older users may come across failed interaction attempts, leading to frustrating interaction experiences and further hindrances to technology use.

It is already known that older adults often struggle with traditional mouse-based "point-and-click" input techniques, with common problems including - but not limited to - the need of cursor relocation, double-clicking, accidental target slip-offs, and click-todrag tasks [3]. In order to avoid transferring these issues to the midair medium, interfaces that make use of point-to-select input techniques should provide additional support for users that experience changes in pointing abilities. However, researchers have yet to identify effective and accessible methods for doing so.

Because mid-air pointing techniques often offer limited to no natural haptic feedback and usually rely solely on unimodal visual feedback through hand-GUI coordination [29], it is pertinent to question if multimodal feedback could support and even improve older adults" "point-and-select" abilities in mid-air. Prior research has suggested possible benefits of providing multimodal feedback for older users in mouse-based "drag-and-drop" tasks [23], touchscreen mobile interaction [22], and gaming [24, 35], but given the "hands-free" and touchless nature of mid-air interaction, the effects of multimodal feedback on older adults' pointing and selection performance in mid-air are still not widely understood.

As "hands-free" interaction starts to appear in a growing number of interaction contexts (motion-based games, smart homes, intelligent car interfaces, virtual and augmented reality, interactive walls and more), it is essential to understand if this novel input method is aligned with the abilities and needs of the growing older population and further explore possibilities for supporting usable and accessible interactions. Therefore, we report the findings of a target acquisition experiment in which older adults had to perform "pointand-select" gestures in mid-air whilst different feedback types were being provided. We compared performance and subjective workload ratings of 6 feedback conditions: visual only, audio only, haptic only, visual-and-audio, visual-and-haptic, and audio-andhaptic. Our results contribute to a better understanding of how feedback modality may improve the usability and accessibility of mid-air gesture interaction for older adults with diverse abilities.

\section{RELATED WORK}

\subsection{Age-related Changes in Pointing Abilities}

The natural course of ageing leads to gradual decline in sensory, cognitive and motor functions $[8,30]$. These natural changes affect how older adults engage with all aspects of daily activities, including computer-mediated tasks [8, 24]. For instance, research has been conducted to better understand the role of age-related changes in mouse aptitude [3], web browsing [34], and touchscreen performance $[11,25]$. It was also observed in older adults a decreased ability of controlling movement amplitudes and scale velocity that contributed to slower, more variable movements within target acquisition tasks [9]. However, older adults' pointing performance in mid-air - including means of supporting it - is a topic yet to be fully addressed and understood. Recent research on how older adults use freehand gestures for TV menu control [20] and computer tasks [7] found empirical evidence that older adults - unlike younger users - indeed struggle at "point-and-select" tasks in mid-air, however this issue was not deeply explored in those studies and the question about how can we design age-friendly midair interactions that support pointing and selection abilities of older users is still unanswered.

There are many ageing factors that compromise the pointing abilities of older users. Muscle strength begins to gradually decline from the age of 50 [18], leading to easy fatiguing, decrease in motor control, limited range of motion and slower reaction times [8, 24]. As a matter of comparison, an older adult at the age of 90 is expected to have a range of motion that is only $60 \%$ of the range of motion of an average 30 years old individual $[8,18]$. Furthermore, continuous use of hand and arm movements for pointing in mid-air, without proper and frequent relaxation of the arms, can lead older users to physical tiredness and may largely impact accuracy and steadiness of movements, performance consistency, and user experience throughout the interaction [7, 24, 33]. Age-related health conditions such as Arthritis and Parkinson's disease may also create further impediments for older individuals $[8,24,30]$.

Gradual decline in sensory and cognitive functions may also affect the pointing abilities of older users in mid-air. Reduced visual perception is a main contributing factor on how older users perceive and interact with different interfaces, specifically touchless interfaces, since most interaction methods rely heavily on visual feedback $[6,11]$. Older adults may face greater difficulties at following cursor movements, locating targets on screen, and perceiving GUI changes due to limited visual acuity, which can also affect reaction times once they make a gesture [18, 20, 30]. Aged hearing function and reduced sensitivity to sound [18] can also affect the effectiveness of sound cues and audio feedback in cases where these are provided. Despite limited haptics in mid-air, reduced tactile sensitivity and acuity may also contribute to poorer "point-and-select" performance. The skin of older individuals 
Evaluating the Effects of Feedback Type on Older Adults'

Performance in Mid-Air Pointing and Target Selection

become less sensitive to pressure after the age of 50 and there is a reduced ability to perceive vibrations and recognise different shapes and textures by touch $[18,30]$. Besides tactile sensitivity, tactile spatial acuity is also affected and may affect tasks requiring orientation and hand dexterity $[11,18]$. Reduced touch and tactile perception need to be taken into account when choosing GUI arrangements and pressure points for interface elements as well as haptic feedback for mid-air interactions [11]. Natural age-related decline in cognitive functions may also be involved in greater efforts for motor learning and motor recall which can jeopardise motor-based interaction in mid-air [8, 18, 24].

\subsection{Uni-and-Multimodal Feedback in Mid-air}

2.2.1 Visual feedback. To date, most gesture-based interfaces rely heavily - and sometimes exclusively - on visual feedback [14]. Visual feedback provides useful support and most users will expect some form of visual information to rely on. For instance, user interfaces based on pointing gestures may provide continuous visual feedback about hand position $[1,10]$ as well as indicate the outcomes of gesture commands on screen [7]. However, unimodal visual feedback can bring some fundamental issues: virtual elements and transitions can be easily occluded by the hand during the course of the interaction [29], feedback may become imperceptible on small screens or at a distance (for large gesturebased interactive walls) [2], and may be inaccessible for users with visual impairments [19]. Age-related decline in visual processing and acuity may also be a contributing obstacle for older users interacting with gesture-based interfaces that exclusively rely on visual feedback $[7,18]$.

2.2.2 Audio feedback. Although not as widely used as visual feedback, audio feedback is mostly used to support visual feedback and indicate whether gesturing in mid-air has been successful [32]. Literature regarding the exploration and effectiveness of audio feedback for gesturing in mid-air is rather limited. In [31], users receive audio feedback after selecting items by tapping on the palm of their hand, while BoomRoom [21] uses real objects to augment audio feedback for emitting sounds after the user gestures in midair. Despite the usefulness of providing audio feedback after the user's input, [14] argue that functional feedback gives no insight into how users are being sensed. Indeed, research has suggested that providing feedback for indicating whether the user's hand is being sensed and whether they are gesturing in the right place can be of great use for older users and should be explored in more depth [7, $15]$.

2.2.3 Haptic feedback. Interaction in mid-air provides limited to no haptic feedback due to its touchless nature. Haptic feedback has been implemented on an experimental level using two methods: through contact-based vibrotactile stimulation $[4,14]$ and through non-contact force [32]. Although the benefits of providing haptic feedback in mouse-based target acquisition tasks $[2,28]$ have been suggested in prior research, the benefits of implementing haptic feedback in mid-air are still not well known. It is also unclear if the inclusion of haptic feedback may improve the performance of older adults in pointing and selection tasks.
SUI '18, October 13-14, 2018, Berlin, Germany

2.2.4 Multimodal feedback. Research has indicated that multimodal feedback improved older adult's performance in completing drag-and-drop tasks on a computer [23] and during touchscreen interaction [22]. Providing multimodal feedback in mid-air may be useful in supporting older users who experience different levels of visual, auditory or tactile processing decline. However, research on the effects of different multimodal feedback combinations has not been widely explored yet.

\subsection{Empirical Studies on Pointing in Mid-air}

Haque et al. [16] described Myopoint, a barehand pointing and clicking technique using forearm mounted electromyography and inertial motion sensors. Myopoint's accuracy and speed were evaluated with young adults using Vogel and Balakrishnan's [13] experiment design that consisted of freehand pointing and clicking tasks on a large display. Winkler et al. [12] investigated the effectiveness of mid-air pointing interaction on projector phones with 12 young adults. Their findings suggested that interaction techniques that integrate touch and mid-air pointing may enrich projector experiences.

Nancel et al. [26, 27] explored mid-air pointing on ultra-walls (wall-sized displays). Novel pointing techniques were designed based on the theoretically assumption that high precision pointing on ultra-walls. All the empirical studies described above contributed to some aspect of advancing mid-air pointing techniques. However, studies involving older users (60+ years old) are still necessary in order to understand if pointing techniques are aligned with the physical abilities of the older population. Older users may be excluded from technology advancements in the field if their physical abilities and preferences are not taken into account. Furthermore, the role of feedback on the performance of mid-air pointing techniques and target selection has not been largely explored yet and might contribute to the usability of pointing interactions for the older population.

\section{EXPERIMENT}

\subsection{Participants}

25 older adults (12 female) participated in the experiment. Participants were aged 60 to 83 (mean age: 67; SD=6.7) with normal-to-corrected vision and had prior computer experience with some familiarity with touchscreen interaction. No prior experience with motion sensing devices and mid-air pointing interaction was reported. Before the start of the experiment, participants had their manual dexterity and motor skills assessed using a Rolyan 9-Hole Peg Toolkit [37], which confirmed that all participants were within the norms for their age group. Two participants were left-handed. The study has been reviewed by the University of Reading's Research Ethics Committee and has been given a favourable ethical opinion for conduct. 


\subsection{Task}

After being introduced to the Leap Motion sensor, participants were made aware that they were able to control the cursor on-screen by moving their hand in mid-air. After understanding the interaction, participants were told they were able to select the target shown on screen by making a pinch gesture once they located the target with the cursor on it (Figure 2). Then, participants were given a practice session of 10 trials and were asked to select the following targets as fast as possible whilst visual feedback was being provided. The practice session served to make participants familiar to the Leap Motion's spatial field of interaction and gesturing in mid-air as well as minimising learning effects for the following sessions.

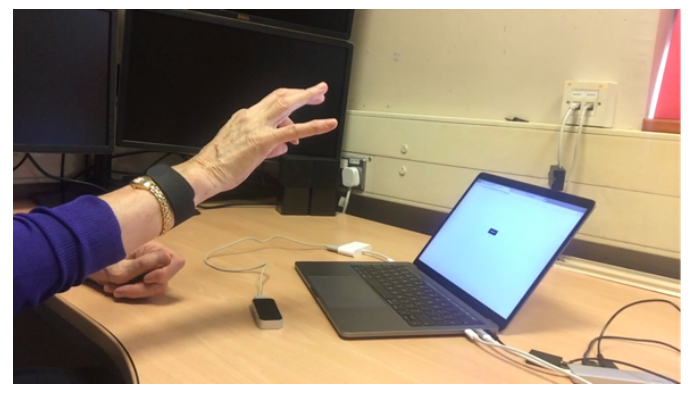

Figure 2: Participant pointing in mid-air and selecting an onscreen target by making a pinch gesture while receiving haptic feedback through a wearable wristband.

After completing the practice session, participants were asked to complete a target acquisition task consisting of 21 targets shown randomly on screen. The first target was not included in the analysis because it usually involved repositioning of the hand and finding a comfortable posture. Participants were asked to locate the target by pointing and select it by making a pinch gesture as fast as possible, while being given feedback. Participants repeated the task for 6 feedback types, 3 were unimodal feedback (Visual or Audio or Haptic) and 3 were multimodal feedback (Visual-and-Audio, Visual-and-Haptic or Audio-and-Haptic). The order was counterbalanced across participants to minimise fatigue and learning effects. After each round, participants were asked to complete a NASA TLX (Task Load Index) questionnaire about the subjective workload of each feedback type.

\subsection{Apparatus}

Participants interacted in a sitting position with a Leap Motion sensor connected to a 13-inch MacBook with built-in retina display at 2560x1600 pixels resolution (227 ppi) as shown in Figure 2.

\subsection{Targets}

Target size was 115 pixels for width and 50 pixels for height. Target location was defined randomly and shown to participants within the on-screen thresholds in Table 1. Target distances were counterbalanced across trials and Index of Difficulty (ID) ranged between 1 and 1.7 for all possible target position combinations in the study [28].
Table 1: Random possible target positions (in pixels). Zero is top left of the screen.

\begin{tabular}{cc}
\hline x-axis & y-axis \\
\hline 50 & 70 \\
600 & 1200 \\
500 & 50 \\
100 & 1100 \\
300 & 650 \\
680 & 40 \\
50 & 1200 \\
200 & 200 \\
660 & 1200 \\
\hline
\end{tabular}

\subsection{Feedback Design}

Feedback was designed to help older adults in point-and-select tasks in mid-air (Figure 3). Feedback was provided when users located a target on-screen and also for as long as users kept the cursor within the target area. After locating the target (first step: point), users were only able to select the target by making a pinch gesture while still hovering it (second step: select). Users were able to see the cursor location on all 6 feedback types. The feedback conditions used in our experiment are explained below:

- Visual Feedback (V): An on-screen target change in colour and contrast is provided when the user locates a target. From grey (hexadecimal \#C2C2D6) to red (\#C70000).

- Audio Feedback (A): A continuous tone cue of 44100 $\mathrm{Hz}$ (32-bit) is provided when the users locates a target.

- Haptic Feedback (H): On-skin vibrotactile feedback is provided through a wearable wristband. The wristband is built with a micro bluetooth subwoofer that provides continuous vibrations at $55 \mathrm{~Hz}$ when users locate a target. The development of the haptic engine was informed by [14] and [4].

- Visual-and-Audio Feedback (VA): A combination of Visual only and Audio only feedback at the same time.

- Visual-and-Haptic Feedback (VH): A combination of Visual only and Haptic only feedback at the same time.

- Audio-and-Haptic Feedback (AH): A combination of Audio only and Haptic only feedback at the same time. 


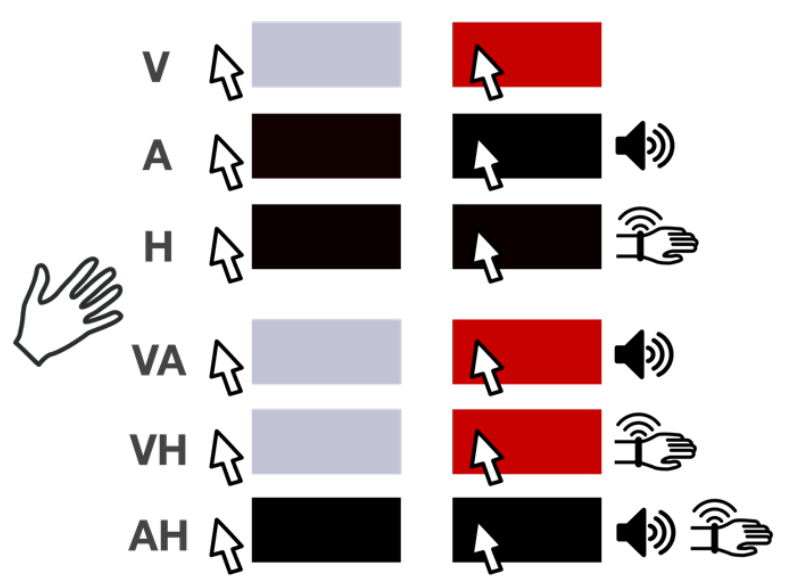

Figure 3: Target selection and feedback type scheme.

\section{RESULTS}

3000 trials (20 targets x 6 feedback conditions x 25 participants) were analysed in the experiment. This session report our findings.

\subsection{Target Selection Time}

Figure 4 shows the average time taken to select a target for each feedback type in mid-air. Target selection time presented great variability across trials, with targets being selected as fast as 1 second and as slow as 1 minute due to multiple target mis-selections and slip-offs. Participants receiving different feedback types achieved average target selection times between 6.2 seconds (Visual-and-Audio feedback) and 7.4 seconds (Visual feedback only). The average selection time across participants - regardless of feedback type - was 6.8 seconds per target. A repeated-measures ANOVA on time to select targets for 6 feedback types showed a significant main effect for feedback type on selection time $[\mathrm{F}(5$, 2994 $)=2.25, p=0.03$ ]. Post-hoc pairwise Tukey HSD tests showed that the time taken to select targets on multimodal Visual-andAudio feedback (6.2 seconds) was significantly lower than the time taken on unimodal Visual feedback ( 7.4 seconds, $\mathrm{p}=0.02$ ) and Audio feedback ( 7.2 seconds, $\mathrm{p}=0.03)$. No significant differences were found for the remaining feedback types $(\mathrm{p}>0.05)$.
Average time for target selection in mid-air for each feedback type provided

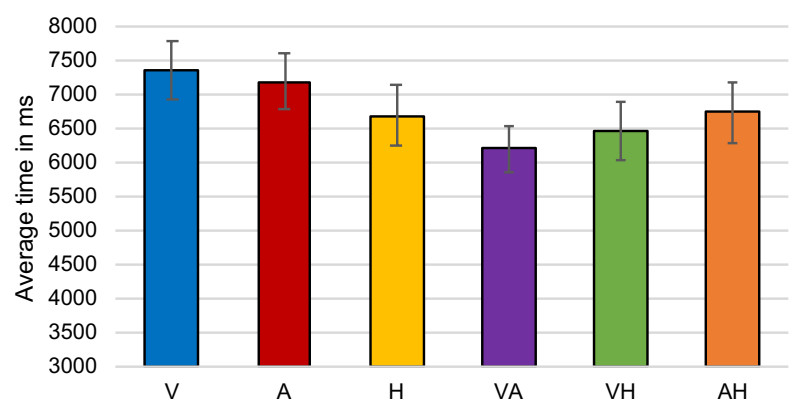

Figure 4: Average time (ms) for selecting on-screen targets $(n=20)$ by making a pinch gesture in mid-air. Error bars represent S.E.

\subsection{Accuracy}

Participants achieved a successful target selection after 1.7 gesture attempts on average (i.e. how many times they had to make a pinch gesture to select a specific target). Average number of target selection attempts ranged between 1.5 attempts on Visual-andAudio feedback (VA) and 1.84 attempts on Visual feedback only (V) (Figure 5). A repeated-measures ANOVA was performed on the number of gesture attempts for target selection and feedback type, however no significant differences were found across all 6 feedback types $[\mathrm{F}(5,5094=2.21, \mathrm{p}=0.39)$.

Average number of target selection attempts in mid-air for each feedback type provided

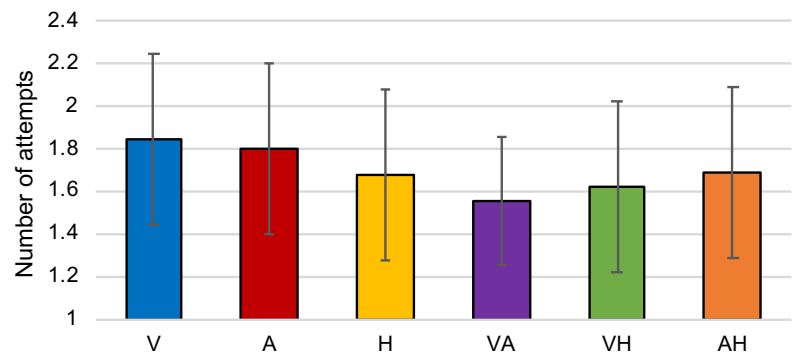

Figure 5: Average number of gesture attempts participants had to make to select a target successfully. Error bars represent S.E.

Although not presenting great issues at cursor relocation from one target to another, older adults presented substantial difficulties at keeping the cursor within the target once they located it. Lack of hand steadiness lead to multiple unintended target slip-offs and mis-selections, especially when participants attempted to make the pinch gesture to select the target (Figure 6). A great variability was observed across participants, but the average number was 1.9 slipoffs per target (i.e. how many times they exited the target without successfully selecting it). A repeated-measures ANOVA was performed on the number of target slip-offs and feedback type, 
however no significant differences were found across all 6 feedback types $(\mathrm{p}>0.05)$.

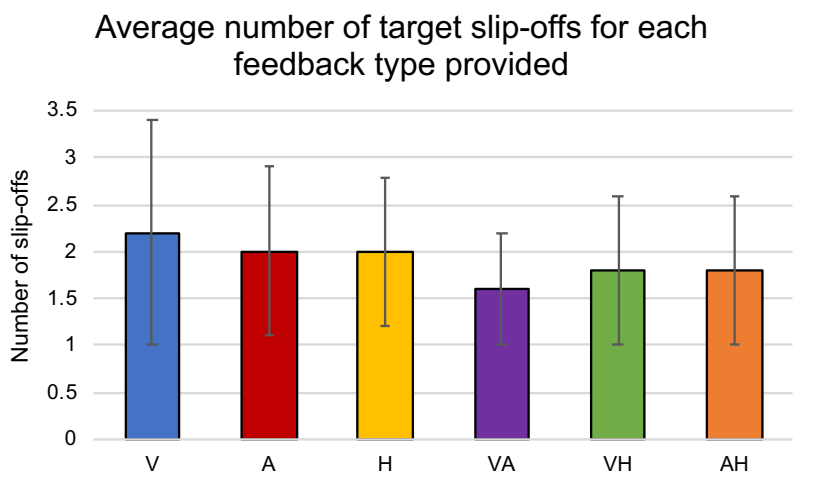

Figure 6: Average number of target slip-offs for each feedback type provided. Error bars represent S.E.

\subsection{Effects of Age}

A two-way repeated-measures ANOVA was performed on the average selection time, comparing the effects of age group (60-69 and $70+)$ and all 6 feedback types $[\mathrm{F}(5,2994)=3.34, \mathrm{p}=0.039]$. Post-hoc pairwise Tukey HSD tests found no significant differences in average selection times among all feedback types provided in the first age group (60-69) [5.5 to 6.3 seconds, $p>$ 0.05]. However, significant differences were found between the average selection time on bimodal Visual-and-Audio feedback (VA) and the remaining 5 feedback types in the older group (70+). That is, selection time on all 6 feedback types were not significantly different for participants in the 60-69 group, but selection time on Visual-and-Audio feedback (VA) was significantly faster (6.4 seconds) than on other feedback types (8.1 to 9.4 seconds) for participants in the $70+$ group $(\mathrm{p}=0.003)$, as shown in Figure 7 .

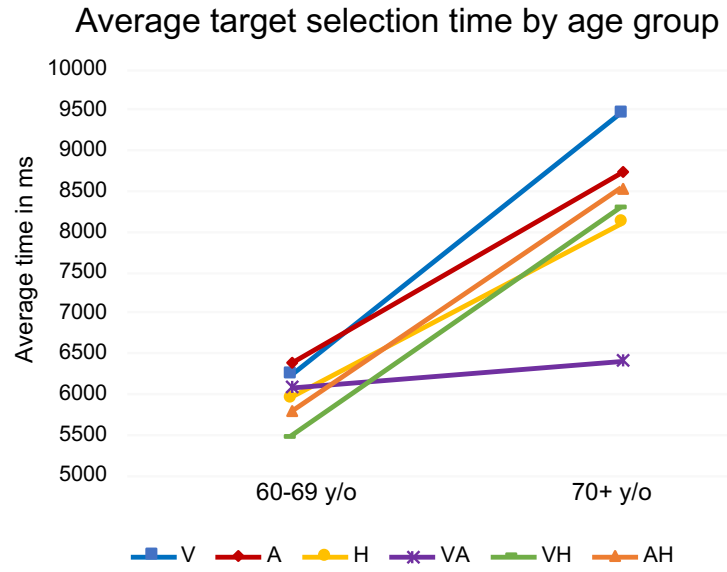

Figure 7: Average target selection time (ms) by age group for each feedback type.

\subsection{Effects of Target Location}

Selection times were isolated by location on-screen as shown in Figure 8. Average times were affected by location, number of target slip-offs and mis-selections but most importantly the number of times participants had to repeat the selection gesture once they located the target. Distance travelled between targets showed no significant effect on time.

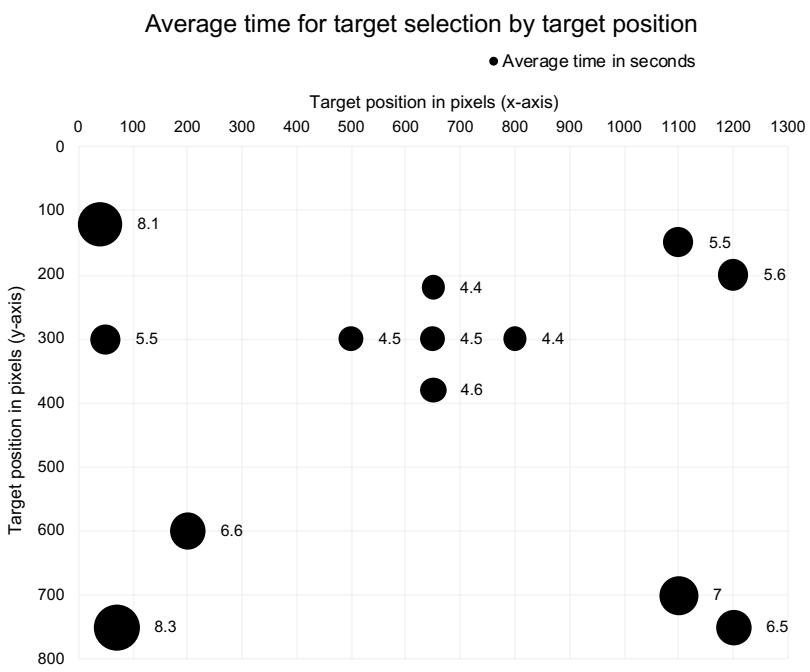

Figure 8: Average target selection time (ms) by target position on-screen (in pixels).

\subsection{Subjective Workload}

The results of the NASA TLX (Task Load Index) questionnaires are shown in Table 2. Scores ranged from 1 (lowest) to 20 (highest). Visual-and-Audio feedback (VA) showed the lowest overall task load index of 9.5 , whereas the other 5 feedback conditions achieved higher scores of 10 and above. An one-way ANOVA was performed on overall task load index scores for all 6 feedback types, however no significant differences were found between all 6 conditions $[\mathrm{F}(5,894)=1.25, \mathrm{p}=0.28)]$.

Further investigation using a two-way ANOVA on the effects of age (60-69 y/o and 70+y/o) on subjective task workload for all 6 feedback conditions found a significant difference between age groups ( $\mathrm{p}=0.028)$. Post-hoc pairwise Tukey HSD tests found no significant differences among overall TLX scores of all 6 feedback conditions in the 60-69 y/o group, however a significant difference was found between the average TLX score for bimodal Visual-andAudio feedback (VA) and the other feedback conditions in the 70+ $\mathrm{y} / \mathrm{o}$ group $(\mathrm{p}=0.02)$. Similar to the results of section 4.3 , participants aged $70+$ found the target acquisition task under bimodal Visualand-Audio feedback (VA) to have a lower subjective workload (overall TLX score of 8.1) in comparison with other feedback conditions (overall TLX scores between 9.2 and 10). 
Evaluating the Effects of Feedback Type on Older Adults'

Performance in Mid-Air Pointing and Target Selection

Table 2: Average scores (1 to 20) from NASA TLX questionnaires for each feedback condition.

\begin{tabular}{rcccccc}
\cline { 2 - 6 } & $\mathbf{V}$ & $\mathbf{A}$ & $\mathbf{H}$ & $\mathbf{V A}$ & $\mathbf{V H}$ & $\mathbf{A H}$ \\
\cline { 2 - 6 } Mental demand & 10 & 9.7 & 10.2 & 8.9 & 9.9 & 9.4 \\
Physical demand & 10.3 & 10 & 9.7 & 9 & 10.1 & 9.6 \\
Temporal demand & 10.7 & 9 & 9 & 8.3 & 8.8 & 8.5 \\
Performance & 12 & 11.6 & 11.2 & 10.9 & 10.7 & 11.3 \\
Effort & 11.3 & 12.4 & 12 & 10.1 & 10.8 & 10.4 \\
Frustration & 11 & 10.8 & 11.4 & 9.8 & 11.4 & 11 \\
\cline { 2 - 7 } Overall TLX & $\mathbf{1 0 . 9}$ & $\mathbf{1 0 . 6}$ & $\mathbf{1 0 . 6}$ & $\mathbf{9 . 5}$ & $\mathbf{1 0 . 3}$ & $\mathbf{1 0}$ \\
\cline { 2 - 6 } & & & & & &
\end{tabular}

Table 2 headings:

V: Visual feedback only / A: Audio feedback only / H: Haptic feedback only / VA: Visual-and-Audio feedback / VH: Visual-and-Haptic feedback / AH: Audio-and-Haptic feedback

\section{DISCUSSION}

\subsection{Effects of feedback type on pointing tasks}

Our findings indicate that the combination of Visual and Audio feedback (VA) lead to faster target selection in comparison with providing only visual or only audio feedback. Visual-and-Audio feedback, however, did not lead to a lower number of gesturing and selection mistakes in mid-air. Multimodal Visual-and-Audio feedback also achieved a lower subjective workload score in comparison with unimodal feedback types. Despite of older adults taking less time for selecting targets on multimodal Visual-andAudio feedback, we were not able to find significant differences between the other 5 feedback types.

Furthermore, the inclusion of haptic feedback in mid-air did not improve older adults' pointing and gesturing performance in our experiment. It is still unclear, however, if feedback type (uni or multimodal) may improve task completion time or error rates for older users in different contexts of mid-air interaction. Research indicated that level of experience [23] and type of task [4] may play a role in the usefulness of different feedback modalities.

User preference varied among participants, unimodal Audio feedback seemed to be preferred over unimodal Visual feedback. Many participants, including a 73-years-old noted that with audio feedback "I could find the target even with my eyes closed, it feels easier". Unimodal Visual feedback, however, received complaints when the participant's hand would occlude their view of the target in a way participants had to frequently change their posture to solve the problem. Furthermore, some participants found the "buzzing" coming from the wristband that provided Haptic feedback to be too disturbing, while others said they were indifferent about the vibration.
SUI '18, October 13-14, 2018, Berlin, Germany

In relation to ageing, some older users may present a higher decline in vision processing, whereas others may present higher declines in auditory or tactile processing, therefore multimodal feedback should be given preference over unimodal feedback in order to minimise the effects of ageing in mid-air pointing tasks.

\subsection{Age}

Age affected time for selecting targets regardless of feedback type provided. Older adults with ages between 60-69 $(n=13)$ selected targets in 6 seconds on average, while older adults aged $70+(n=7)$ needed 8.2 seconds on average to make a successful target selection. Decreased movement control and pointing steadiness as well as more frequent target slip-offs were observed in the older group. Upper arm fatigue was also noted by some participants regardless of their age, and this issue reflected on the subjective workload responses (NASA TLX) as participants judged that pointing in mid-air required high physical demand (average score of 12.3 out of 20, across all feedback types). Issues with the Leap Motion not being able to recognise the pinch gesture for selecting targets was also judged by older adults as "more frustrating" than pointing and locating a target. Our findings indicated that minimising the need of constant or repetitive gesturing plays a more important role for the usability of pointing interfaces than the feedback type provided. Therefore, we suggest that future work could possibly explore more efficient and age-friendly "selection gesture" options for point-and-select tasks.

\subsection{Target Location}

On-screen target location affected both the selection time and accuracy of target selection by older adults (Figure 8). Targets located on the bottom (left and right) and top left of the screen were highly problematic, leading to more slip-offs and mis-selections than targets located in other coordinates. This issue was possibly due to older adults leaving the Leap Motion sensor's field of view when trying to select those targets (i.e. placing their hand too high or too forward). Instead of moving their hand left-and-right and upand-down just above the sensor, older adults would try to reach the screen and place their hand forward to a point where the hand would leave the Leap Motion's field of view. Participants expressed frustration when trying to reach those targets unsuccessfully. A 80-years-old participant said "my shoulder started to get uncomfortable trying to reach that target on the bottom left, it did not seem to like me". Targets located on the central area and top right of the screen, however, did not present as many issues as the top left and bottom areas. Effects of hand laterality were also not observed. Therefore, based on older users' behavior and sensor capabilities, we suggest that targets should be placed within central area or top right of the screen to ensure that mid-air point-and-select input methods are age-friendly. Screen areas in red (Figure 9) should be avoided. 


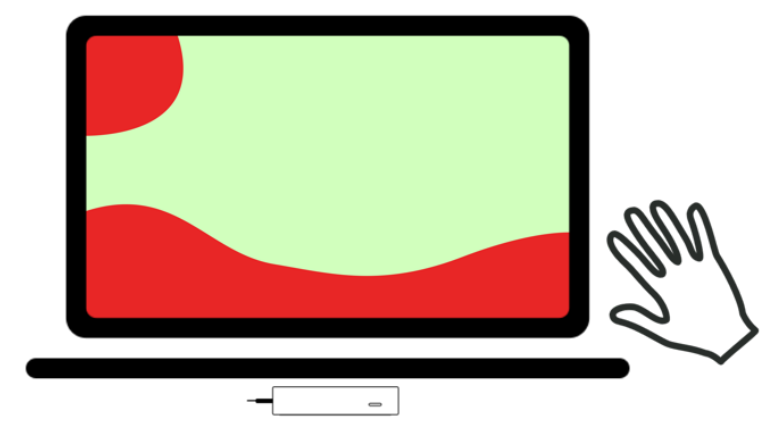

Figure 9: Representation of on-screen areas to avoid placing targets for point-and-select tasks in mid-air (in dark red), whereas areas in bright green should be chosen for age-friendly interfaces.

\section{CONCLUSION}

In this work, we report the findings of a target acquisition experiment that investigated the effects of different 6 feedback conditions on how older adults performed point-and-select tasks in mid-air. Combined bimodal Visual-and-Audio feedback lead to faster target selection (6.2 seconds on average) in comparison to unimodal Visual or Audio feedback (7.4 and 7.2 seconds respectively). Mid-air point-and-select tasks on bimodal Visualand-Audio feedback also achieved a lower subjective workload among participants aged 70 and older. Effects of other feedback combinations on target selection time were not significant. Accuracy of pointing and selection gestures were not affected by feedback modality. Furthermore, target location on screen showed to be a more decisive factor for older adults' pointing and selection performance in mid-air than feedback modality. Our findings contribute to the advancement of mid-air pointing techniques and to a better understanding of how feedback modality may improve the usability and accessibility of mid-air gesture interaction for older users who experience changes in pointing abilities.

\section{ACKNOWLEDGMENTS}

We thank all the volunteers for their participation and the University of Reading's Hugh Sinclair Unit for their help with recruitment. The research is funded by the Brazilian Science Without Borders Programme, an initiative under the Ministry of Education and CAPES Foundation. Project number BEX 1303713-7.

\section{REFERENCES}

[1] Alex Butler, Shahram Izadi, and Steve Hodges. 2008. SideSight: multi"touch" interaction around small devices. In Proceedings of the 21st annual ACM symposium on User interface software and technology (UIST '08). ACM, New York, NY, USA, 201-204. DOI: https://doi.org/10.1145/1449715.1449746

[2] Andy Cockburn and Stephen Brewster. 2005. Multimodal feedback for the acquisition of small targets. Ergonomics. 48, 9, 1129-1150.

[3] Anna Dickinson, Roos Eisma, and Peter Gregor. 2011. The barriers that older novices encounter to computer use. Universal Access in the Information Society. 10, 3, 261-266. DOI: https://doi.org/10.1007/s10209-010-0208-6
[4] Anne Köpsel, Päivi Majaranta, Poika Isokoski, Anke Huckauf. 2016. Effects of auditory, haptic and visual feedback on performing gestures by gaze or by hand. Behaviour \& Information Technology. 35, 12: 1044-1062.

[5] Aqueasha Martin-Hammond, Foad Hamidi, Tejas Bhalerao, Abdullah Ali, Catherine Hornback, Casey Means, and Amy Hurst. 2017. The Participatory Design of an Adaptive Interface to Support Users with Changing Pointing Ability. In Proceedings of the 19th International ACM SIGACCESS Conference on Computers and Accessibility (ASSETS '17). ACM, New York, NY, USA, 343-344. DOI: https://doi.org/10.1145/3132525.3134810

[6] Arthur Theil Cabreira and Faustina Hwang. 2015. An analysis of mid-air gestures used across three platforms. In Proceedings of the 2015 British HCI Conference (British HCI '15). ACM, New York, NY, USA, 257-258. DOI: http://dx.doi.org/10.1145/2783446.2783599

[7] Arthur Theil Cabreira and Faustina Hwang. 2016. How Do Novice Older Users Evaluate and Perform Mid-Air Gesture Interaction for the First Time?. In Proceedings of the 9th Nordic Conference on Human-Computer Interaction (NordiCHI '16). ACM, New York, NY, USA. DOI: https://doi.org/10.1145/2971485.2996757

[8] Caroline J. Ketcham and George E. Stelmach. 2001. Age-Related Declines in Motor Control. In Birren, J.E. \& Schaie, K.W. (Eds.), Handbook of the Psychology of Aging. Academic Press.

[9] Caroline J. Ketcham, Rachael D. Seidler, Arend W.A. Van Gemmert, and George E. Stelmach. 2002. Age-Related Kinematic Differences as Influenced by Task Difficulty, Target Size, and Movement. The Journals of Gerontology: Series B. 57, 1, 54-64.

[10] Chris Harrison and Scott E. Hudson. 2009. Abracadabra: wireless, highprecision, and unpowered finger input for very small mobile devices. In Proceedings of the 22nd annual ACM symposium on User interface software and technology (UIST '09). ACM, New York, NY, USA, 121-124. DOI: https://doi.org/10.1145/1622176.1622199

[11] Christian Stößel. 2009. Familiarity as a factor in designing finger gestures for elderly users. In Proceedings of the 11th International Conference on Human-Computer Interaction with Mobile Devices and Services (MobileHCI '09). DOI: http://dx.doi.org/10.1145/1613858.1613950

[12] Christian Winkler, Ken Pfeuffer, Enrico Rukzio. 2012. Investigating mid-air pointing interaction for projector phones. In Proceedings of the 2012 ACM international conference on Interactive tabletops and surfaces (ITS '12), 8594. DOI: https://doi.org/10.1145/2396636.2396650

[13] Daniel Vogel and Ravin Balakrishnan. 2005. Distant freehand pointing and clicking on very large, high resolution displays. In Proceedings of the 18th annual ACM symposium on User interface software and technology (UIST '05), 33-42. DOI: http://dx.doi.org/10.1145/1095034.1095041

[14] Euan Freeman, Stephen Brewster, and Vuokko Lantz. 2014. Tactile Feedback for Above-Device Gesture Interfaces: Adding Touch to Touchless Interactions. In Proceedings of the 16th International Conference on Multimodal Interaction (ICMI '14). ACM, New York, NY, USA, 419-426. DOI: https://doi.org/10.1145/2663204.2663280

[15] Euan Freeman, Stephen Brewster, and Vuokko Lantz. 2016. Do That, There: An Interaction Technique for Addressing In-Air Gesture Systems. In Proceedings of the 2016 CHI Conference on Human Factors in Computing Systems (CHI '16). ACM, New York, NY, USA, 2319-2331. DOI: https://doi.org/10.1145/2858036.2858308

[16] Faizan Haque, Mathieu Nancel, Daniel Vogel. 2015. Myopoint: Pointing and Clicking Using Forearm Mounted Electromyography and Inertial Motion Sensors. In Proceedings of the 33rd Annual ACM Conference on Human Factors in Computing Systems (CHI '15), 3653-3656.

[17] Faustina Hwang, Simeon Keates, Patrick Langdon, and P. John Clarkson. 2003. Multiple haptic targets for motion-impaired computer users. In Proceedings of the SIGCHI Conference on Human Factors in Computing Systems (CHI '03). ACM, New York, NY, USA, 41-48. DOI: http://dx.doi.org/10.1145/642611.642620

[18] Felicia Huppert. 2003. Designing for Older Users. In: P. John Clarkson, Roger Coleman, Simeon Keates, and Cherie Lebbon (Eds.), Inclusive 
Evaluating the Effects of Feedback Type on Older Adults' Performance in Mid-Air Pointing and Target Selection

Design: Design for the whole population. Springer, London. DOI https://doi.org/10.1007/978-1-4471-0001-0_2

[19] Holly S. Vitense, Julie A. Jacko, and V. Kathlene Emery. 2002. Multimodal feedback: establishing a performance baseline for improved access by individuals with visual impairments. In Proceedings of the fifth international ACM conference on Assistive technologies (Assets '02). ACM, New York, NY, USA, 49-56. DOI: http://dx.doi.org/10.1145/638249.638260

[20] Jan Bobeth, Susanne Schmehl, Ernst Kruijff, Stephanie Deutsch, and Manfred Tscheligi. 2012. Evaluating performance and acceptance of older adults using freehand gestures for TV menu control. In Proceedings of the 10th European Conference on Interactive TV and Video (EuroITV '12). ACM, New York, NY, USA, 35-44. DOI: https://doi.org/10.1145/2325616.2325625

[21] Jörg Müller, Matthias Geier, Christina Dicke, and Sascha Spors. 2014. The boomRoom: mid-air direct interaction with virtual sound sources. In Proceedings of the SIGCHI Conference on Human Factors in Computing Systems (CHI '14). ACM, New York, NY, USA, 247-256. DOI https://doi.org/10.1145/2556288.2557000

[22] Ju-Hwan Lee, Ellen Poliakoff, and Charles Spence. 2009. The Effect of Multimodal Feedback Presented via a Touch Screen on the Performance of Older Adults. In Proceedings of the 4th International Conference on Haptic and Audio Interaction Design (HAID '09). Springer, Berlin, Heidelberg, 128135. DOI: https://doi.org/10.1007/978-3-642-04076-4 14

[23] Julie Jacko, V. Kathlene Emery, Paula J. Edwards, Mahima Ashok, Leon Barnard, Thitima Kongnakorn, Kevin P. Moloney, and François Sainfort. 2004. The effects of multimodal feedback on older adults' task performance given varying levels of computer experience. Behaviour \& Information $\begin{array}{lllll}\text { Technology. } & 23, & 4, & 247-264 & \text { DOI: }\end{array}$ https://doi.org/10.1080/01449290310001659213

[24] Kathrin Gerling, Ian Livingston, Lennart Nacke, and Regan Mandryk. 2012. Full-body motion-based game interaction for older adults. In Proceedings of the SIGCHI Conference on Human Factors in Computing Systems (CHI '12), 1873-1882. DOI: http://dx.doi.org/10.1145/2207676.2208324

[25] Leah Findlater, Jon E. Froehlich, Kays Fattal, Jacob O. Wobbrock, and Tanya Dastyar. 2013. Age-related differences in performance with touchscreens compared to traditional mouse input. In Proceedings of the SIGCHI Conference on Human Factors in Computing Systems (CHI '13). ACM, New York, NY, USA, 343-346. DOI: https://doi.org/10.1145/2470654.2470703

[26] Mathieu Nancel, Emmanuel Pietriga, Olivier Chapuis, Michel BeaudouinLafon. 2015. Mid-Air Pointing on Ultra-Walls. ACM Trans. Comput.-Hum. Interact. 22, 5. DOI: https://doi.org/10.1145/2766448

[27] Mathieu Nancel, Julie Wagner, Emmanuel Pietriga, Olivier Chapuis, Wendy Mackay. 2011. Mid-air pan-and-zoom on wall-sized displays. In Proceedings of the SIGCHI Conference on Human Factors in Computing Systems (CHI '11), 177-186. DOI: https://doi.org/10.1145/1978942.1978969

[28] Motoyuki Akamastu, I. Scott MacKenzie, Thierry Hasbrouc. 1995. A comparison of tactile, auditory, and visual feedback in a pointing task using a mouse-type device. Ergonomics, 38, 816-827.

[29] Peng Song, Wooi Boon Goh, William Hutama, Chi-Wing Fu, and Xiaopei Liu. 2012. A handle bar metaphor for virtual object manipulation with midair interaction. In Proceedings of the SIGCHI Conference on Human Factors in Computing Systems (CHI '12). ACM, New York, NY, USA, 1297-1306. DOI: $\underline{\text { http://dx.doi.org/10.1145/2207676.2208585 }}$

[30] Sara J. Czaja and Chin Chin Lee. 2007. Information technology and older adults. In J. A. Jacko \& A. Sears (Eds.), The Human Computer-Interaction Handbook (2nd ed., 777-792. Lawrence Erlbaum Associates, New York.

[31] Sean Gustafson, Christian Holz, and Patrick Baudisch. 2011. Imaginary phone: learning imaginary interfaces by transferring spatial memory from a familiar device. In Proceedings of the 24th annual ACM symposium on User interface software and technology (UIST '11). ACM, New York, NY, USA, 283-292. DOI: https://doi.org/10.1145/2047196.2047233

[32] Tom Carter, Sue Ann Seah, Benjamin Long, Bruce Drinkwater, and Sriram Subramanian. 2013. UltraHaptics: multi-point mid-air haptic feedback for
SUI '18, October 13-14, 2018, Berlin, Germany

touch surfaces. In Proceedings of the 26th annual ACM symposium on User interface software and technology (UIST '13). ACM, New York, NY, USA, 505-514. DOI: https://doi.org/10.1145/2501988.2502018

[33] Vanessa Vuibert, Wolfgang Stuerzlinger, and Jeremy R. Cooperstock. 2015. Evaluation of Docking Task Performance Using Mid-air Interaction Techniques. In Proceedings of the 3rd ACM Symposium on Spatial User Interaction (SUI '15). ACM, New York, NY, USA, 44-52. DOI: https://doi.org/10.1145/2788940.2788950

[34] Vicki L. Hanson and Susan Crayne. 2005. Personalization of Web browsing: adaptations to meet the needs of older adults. Universal Access in the Information Society. 4, 1, 46-58. DOI: https://doi.org/10.1007/s10209-0050110-9

[35] Wijnand Ijsselsteijn, Henk Herman Nap, Yvonne de Kort, and Karolien Poels. 2007. Digital game design for elderly users. In Proceedings of the 2007 Conference on Future Play (Future Play '07). ACM, New York, NY, USA, 17-22. DOI: http://dx.doi.org/10.1145/1328202.1328206

[36] Wei Lu, Zheng Tong, Jinghui Chu. 2016. Dynamic Hand Gesture Recognition With Leap Motion Controller. IEEE Signal Processing Letters. 23, 9: 1188-1192.

[37] Ying Chih Wang, Richard W. Bohannon, Jay Kapellusch, Arun Garg, Richard C. Gershon. 2015. Dexterity as measured with the 9-Hole Peg Test (9-HPT) across the age span. Journal of Hand Therapy. 28, 1, 53-60. 